\title{
Suppressive Antiviral Therapy
}

National Cancer Institute

\section{Source}

National Cancer Institute. Suppressive Antiviral Therapy. NCI Thesaurus. Code C155924.

Ongoing antiviral therapy to reduce the frequency of recurrence or prevent transmission. 